\title{
ABNORMAL GRAIN GROWTH IN THREE DIMENSIONS
}

\author{
G.S. Grest ${ }^{1}$, M.P. Anderson ${ }^{1}$, D.J. Srolovitz ${ }^{2}$ and A.D. Rollett ${ }^{3}$ \\ ${ }^{1}$ Corporate Research Science Laboratory, Exxon Research and Engineering Company, \\ Annandale, NJ 08801 USA \\ 2Department of Materials Science and Engineering, University of Michigan, Ann Arbor, MI 48109 USA \\ ${ }^{3}$ Los Alamos National Laboratory, Los Alamos, NM 87545 USA \\ (Received January 16, 1990) \\ (Revised January 30, 1990)

\section{Introduction}

Normal grain growth is characterized by the self-similar coarsening of the microstructure. Therefore, knowledge of the microstructure at one time and the temporal evolution of the mean grain size, $\langle R\rangle$, provides all of the information required for a complete statistical description of the evolving microstructure. In contrast, abnormal grain grow:h is characterized by the growth of a small number of grains at a rate in excess to that of the mean grain size and, consequently, a lack of self-similarity. Abnormal grain growth is most frequently observed in samples containing a dispersed second phase and/or in sheet materials.

An early attempt at analyzing abnormal grain growth suggested that if a grain was larger than the largest grain in the steady-state normal grain size distribution, that grain would grow abnormally [1]. Two-dimensional Monte Carlo computer simulations have shown [2] that such large grains grow, but at a slower rate than the mean grain size - i.e., the large grains become incorporated in the steady-state normal grain size distribution function. Reanalysis of the theoretical basis for this picture of abnormal grain growth yields results consistent with the simulation results [3].

Additional two dimensional simulations have been performed to investigate other possible explanations of abnormal grain growth. Monte Carlo simulations have shown that anisotropy in grain boundary energy can lead to the abnormal growth of grains with grain boundary energies much smaller than the grain boundary energy of "normal" grains. However, this abnormal growth process occurs by a wetting phenomenon which yields microstructures which are rather different than those typically observed experimentally. Both Monte Carlo and front tracking methods have been applied to study the effects of anisotropy in grain boundary mobility on abnormal grain growth $[4,5]$. Such anisotropy in grain boundary mobility may be attributable to the natural variation in mobility with grain boundary structure and may be further enhanced by a pre-existing texture (this is similar to the concept of "oriented growth" in recrystallization). These studies show that large grains with high grain boundary mobilities (relative to the "normal" grains) do indeed grow abnormally. A simple theoretical analysis shows that the ratio of the sizes of the abnormal grains to the mean "normal" grain size saturates at long time to a mobility dependent constant [4]. Nonetheless, these ratios can be rather large and abnormal grains may impinge and consume the entire "normal" matrix prior to achieving the asymptotic grain size.

The purpose of the present study is to re-examine the effect of grain boundary mobility anisotropy on abnormal growth for the more realistic case of grain growth in three dimensions.

\section{Simulation Procedure}

The simulation procedure employed in the present study is similar to that used in previous studies of normal grain growth in three dimensions [6]. The grain structure is represented on a simple cubic lattice consisting of either $\mathrm{N}=100 \times 100 \times 100$ or $\mathrm{N}=140 \times 140 \times 140$ sites. Each lattice site is assigned a number $(1-\mathrm{Q}$, where $\mathrm{Q}=48)$ corresponding to a particular grain orientation. The bond between neighbor sites is assigned a positive energy if the sites have different orientation (i.e., a grain boundary bond) and zero if the sites connected by the bond are alike. In the present simulation, a site interacts with all neighbors out to a distance of $\sqrt{3} \mathrm{a}_{0}$, where $\mathrm{a}_{0}$ is the simple cubic 
(a)

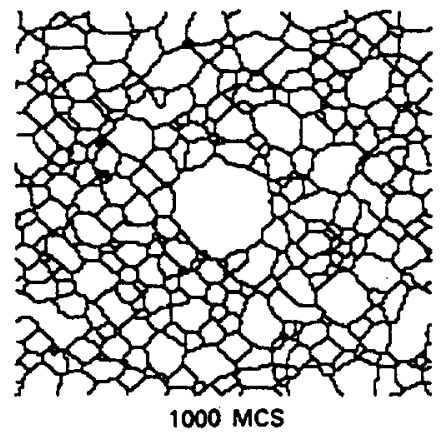

(b)

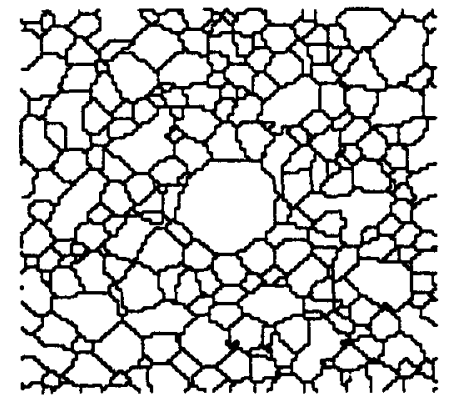

1000 MCs

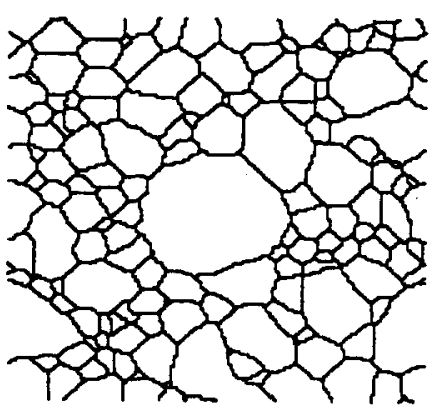

5000 MCS

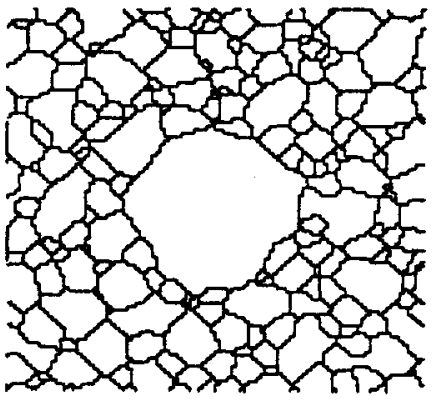

5000 MCS
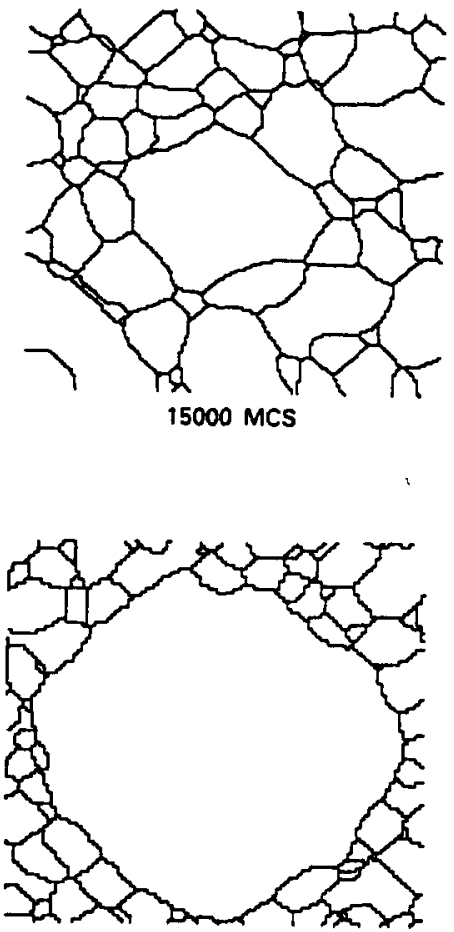

15000 MCS

Fig. 1 The temporal evolution of the central cross section of pre-grown normal grain growth microstructures in which large spherical grains were inscribed at 0 MCS. The microstructure in (a) initially had $V_{1} /\langle V\rangle=50$ on a $140^{3}$ lattice and $\mu=1$, while the microstructure in (b) initially had $V_{1} /\langle V\rangle=15$ on a $100^{3}$ lattice and $\mu=10$.

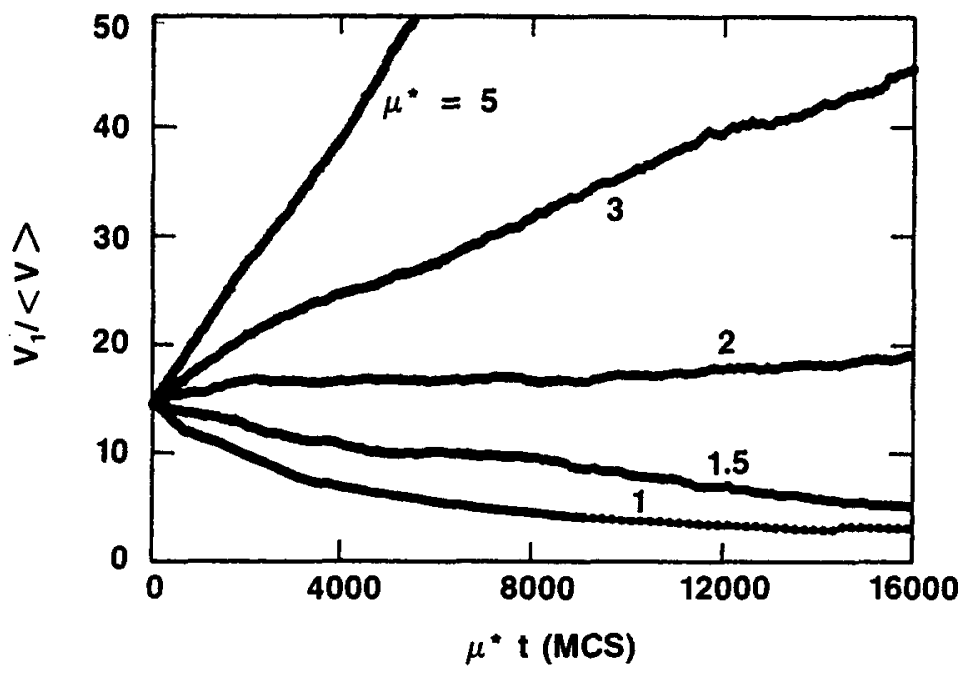

Fig. 2 The ratio of the volume of the large central grain to the mean normal grain volume $\left.\left(V_{1} /<V\right\rangle\right)$ as a function of time for $\mu=1.0,1.5,2.0,3.0$ and 5.0. The initial value of $V_{1} /\langle V\rangle$ was 15 for all cases. 
lattice parameter. The kinetics of boundary migration were simulated by a Monte Carlo technique in which a site is selected at random and reoriented to a randomly chosen orientation between 1 and $Q$. If the energy change due to reorientation is less than or equal to zero, the reorientation attempt was accepted. N reorientation attempts is defined as 1 Monte Carlo Step (MCS), which is the unit of time in the present simulations (see Ref. 6 for details).

In order to generalize this method to include grain boundary mobility anisotropy (see Ref. 4 for details), the grain boundaries were divided into two classes: normal and abnormal. If a site, chosen at random, is adjacent to an abnormal grain boundary, a reorientation attempt is made with probability one. If the chosen site is adjacent to a normal grain boundary, a reorientation attempt is made with probability $\mu(\mu \leq 1)$. Two types of simulations were performed. First, a spherical grain was inscribed in the center of a normal 3-d grain growth microstructure (generated in a 1000 MCS normal grain growth simulation yielding $~ 3500$ grains with a mean grain volume $\langle V\rangle=288$ sites - see Ref. 6). The grain boundaries separating this central grain from all of the remaining grains had a mobility advantage $\mu$ over all other grain boundaries. Additional simulations were performed in which a predetermined fraction of randomly chosen grains were assigned high mobility grain boundaries.

\section{$\underline{\text { Results }}$}

Figures $1 \mathrm{a}$ and $\mathrm{b}$ show the temporal evolution of pre-grown normal grain growth microstructures in which large spherical grains (volume $=V_{1}$ ) were inscribed at $0 \mathrm{MCS}$ and which have mobility advantages of $\mu=1$ (i.e. no mobility advantage) and 10 , respectively. While in both cases the large central grains grow, the grain with the higher mobility (Fig. 1b) grows much more quickly. The size of the normal grains appears not to be affected by the presence of the nearby large central grain in either the $\mu=1$ or 10 cases. Furthermore, a comparison of the large central grain with the mean normal grain size $<V>$ shows that the normal, matrix grains are growing faster than the central grain when $\mu=1$ but slower than the central grain when $\mu=10$. Therefore, we conclude that abnormal grain growth is not occurring in the $\mu=1$ (no mobility advantage) case, but is occurring in the $\mu=10$ case.

The effects of the boundary mobility on the growth of the large central grain are summarized in Fig. 2 where we plot the ratio of the volume of the large central grain to the mean normal grain volume $\left(V_{1} /\langle V\rangle\right)$ as a function of time for a range of $\mu$ values. For $\mu>2$, the large central grain grows at a faster rate than the mean normal grain size; i.e., abnormal growth is occurring. No well defined saturation of $V_{1} /\langle V\rangle$ at long times is observed. The effect of initial grain size on abnormal grain growth is indicated in Fig. 3 where the ratio $V_{1} /<V>$ is plotted as a function of time for three initial values of $\left.V_{1} /<V\right\rangle$ between 5 and 27 for $\mu=3$. Clearly, increasing the size of the grain with high grain boundary mobility increases its rate of growth. For the smallest of the central grains $\left[V_{1}(t=0) /<V>\right]$ studied, $V_{1} /<V>$ initially shows a small increase but then appears to saturate at a relatively small value $(-8)$.

Abnormal growth in a more realistic microstructure is shown in Fig. 4 for a mobility ratio of $\mu=10$ and where initially $-3 \%$ of the grains had high mobility grain boundaries. The small grains with high mobility boundaries shrink rapidly and disappear from the structure. The larger grains, with high mobility boundaries, grow into the surrounding "normal" grains and clearly grow at a rate faster than the mean "normal" grain size. The appearance of "new" abnormal grains in the microstructures of Fig. 4 are attributable to the impingement of abnormal grains from below (or above) the plotted cross-section. At late times, the grains with high mobility boundaries impinge upon each other, thereby destroying the intervening "normal" grains. Subsequent coarsening of the structure occurs by normal grain growth. The time dependence of the volume fraction of the material with high mobility boundaries is shown in. Fig. 5. The shape of this curve is well fitted by the Johnson-Mehl-AvramiKolmogorov equation with an Avrami exponent of 3.

\section{Discussion}

Overall, the three dimensional abnormal grain growth simulations are consistent with results previously obtained for two dimensions [4]. Grains with high mobility grain boundaries evolve more quickly than the "normal" grains. As in normal grain growth, grains with high mobility boundaries shrink away if they are small and grow when they are large. Unlike the normal grain growth case, though, large grains with high mobility boundaries grow to sizes much larger than they would if their mobilities were identical to that of the "normal" grains.

The simple abnormal grain growth theory presented in Ref. 4, which was based on earlier work by Hillert [1], provides a basis for the analysis of the growing abnormal grain simulation data. His abnormal grain growth theory predicts the ratio $(\rho)$ of the radius of the grains with high boundary mobility to the mean radius of the "normal" grains $\langle r\rangle$ is given by 


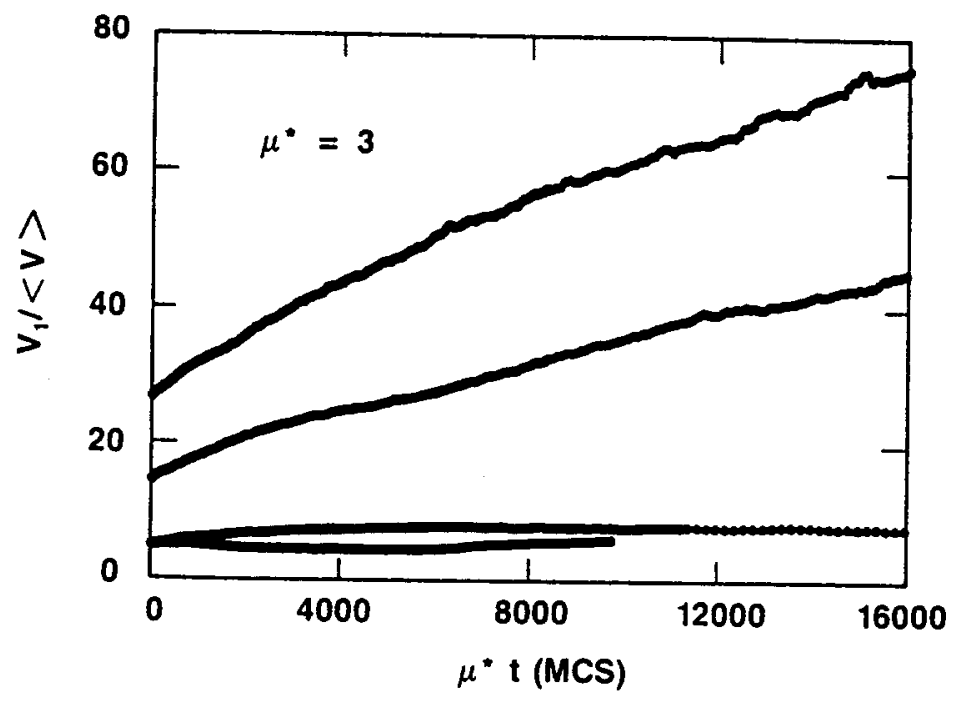

Fig. 3 The ratio of the volume of the large central grain to the mean normal grain volume $\left(V_{1} /\langle V\rangle\right)$ as a function of time for three different initial central grain sizes for $\mu=3$. The initial value of $V_{1} /<V>$ from top to bottom were 27,15 and 5 . In the last case, data for two runs are shown.

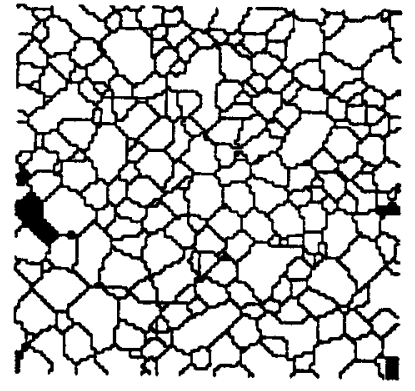

1000 MCS

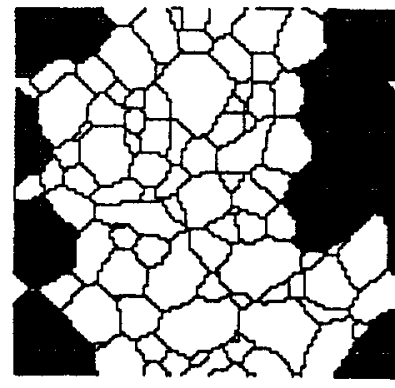

20,000 MCS

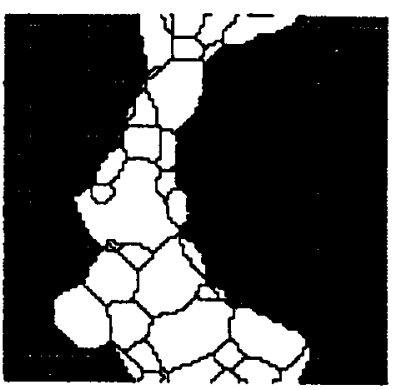

30,000 MCS

Fig. 4 The temporal evolution of a cross-section of an initially normal grain growth microstructure in which $3 \%$ of the grains (randomly chosen) were assumed to have high mobility grain boundaries. The grain boundaries of the shaded grains were assigned a mobility advantage of $\mu=10$.

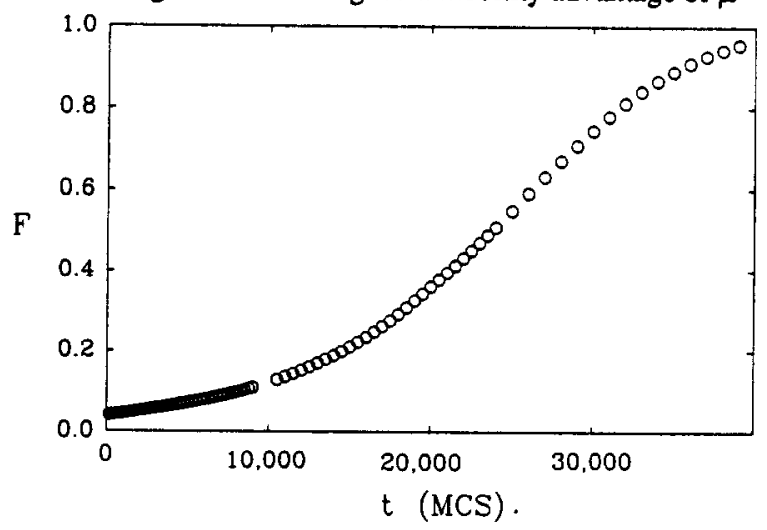

Fig. 5 The volume fraction of grains with high mobility boundaries, F, as a function of time for an entire 3-d microstructure, a cross-section of which was shown in Fig. 4 . 


$$
\frac{d \rho}{d t}=\frac{\gamma M}{2 \rho\langle r\rangle^{2}}\left[\mu(\rho-1)-\rho^{2}\right]
$$

where $\gamma$ is the grain boundary energy per unit area and $M$ is the mobility of the "normal" grain boundaries. Setting the time derivative of $\rho$ to zero, the steady state value of $\rho\left(\rho^{s s}\right)$ was found to be

$$
\rho_{ \pm}^{\text {ss }}=2 \mu[1 \pm \sqrt{1 \cdot 1 / \mu}]
$$

These equations may be interpreted as follows: a grain with high mobility boundaries will be incorporated in the normal distribution if $\rho<\rho_{\text {ss }}^{\text {ss }}, \rho$ will increase to $\rho_{+}$ss for $\rho$.ss $<\rho<\rho_{+}$ss, and $\rho$ will decrease to $p_{+}$ss if $\rho$ is initially greater than $\rho_{+} s s$. Since $\rho_{+} s s>1$ for $\mu>1$, a grain with high mobility boundaries may be incorporated into the normal grain growth grain size distribution function even if it is bigger than the mean grain size. Unfortunately, the relatively small size of the simulation lattice makes it nearly impossible for the grains with high mobility boundaries to achieve their steady state values of $p$ prior to reaching the edges of the simulation cell (e.g. for $\mu=3$, $\rho_{+} s s \approx 11$ and $\left.V_{1}(t=\infty) /<V>=1300\right)$. Nonetheless, the simulations allow us to test a number of other predictions of the theory.

Figure 2 shows that the rate of growth of $\rho$ increases with increasing $\mu$ at fixed initial value of $\rho$, consistent with Eq. (1). Figure 3 shows that the larger the initial value of $\rho$ the larger its rate of increase for fixed $\mu$. Differentiation of Eq. (1) with respect to $\rho$ shows that this rate should increase with increasing $\rho$ for $\rho$. ss $<\rho<2 \sqrt{\mu}$. Since the simulation data shown in Fig. 3 are for initial values of $\rho$ between 1.7 and 2.9 which is less than $2 \sqrt{\mu}=2 \sqrt{3} \approx 3.5$, this result is consistent with the theory. In the $V_{1} /<V>=5(\rho=1.7), \mu=3$ case, the grain with the high mobility boundary initially increases and then appears to saturate at long times. It is unclear at this point whether: (1) the curve is in fact rising very slowly, (2) this behavior is an artifact of the simulation, or (3) the theoretical estimate of $\rho .5 s$ is in error. However, since in the case $\mu=1.5$, the central grain size decays relative to the mean normal grain size (Fig. 2) in contradiction to the theory, we believe that although the theoretical results properly predict the trends seen in the simulations, the theory is not sufficiently quantitative. This conclusion may be attributable to known inadequacies in the original grain growth theory [1] and is consistent with the observations made for the 2-d simulations.

The present results collectively indicate that abnormal grain growth may be attributed to anisotropy in grain boundary mobility, as originally suggested based upon two dimensional simulations [2]. The presence of such anisotropy in experimental systems is generally a consequence of preferred grain orientation (i.e. texture) or the effect of solutes on grain boundary mobility. An experimental result which remains unexplained is the common observation of abnormal grain growth in alloys containing a distribution of precipitates when the material is heat treated near the solvus. Since the precipitates are going into solution, the presence of abnormal growth under these conditions may be associated with solute effects on boundary mobility.

\section{References}

1. M. Hillert, Acta Metall. 13, 227 (1965).

2. D.J. Srolovitz, G.S. Grest and M.P. Anderson, Acta Metall. 33, 2233, (1986).

3. C.V. Thompson, H.J. Frost and F. Spaepen, Acta Metall. 35, 887 (1987).

4. A.D. Rollett, D.J. Srolovitz and M.P. Anderson, Acta Metall. 37, 1227 (1989).

5. H.J. Frost and C.V. Thompson, Acta Metall. 35, 529 (1987).

6. M.P. Anderson, G.S. Grest and D.J. Srolovitz, Phil. Mag. B59, 293 (1989). 